\title{
Effect of ratio oil to potato on amount of oil uptake and quality of potato French fries
}

\begin{abstract}
In deep fat fried products health should be addressed to meet consumer demand the soft and moist interior together with crispy crust are desirable characteristics of most fried food. In this study the effect of ratio oil to potato on amount of oil uptake of potato strips at two size were investigated. Results showed that ratio oil to potato 20:1 lead to increase in potato loss of strips during frying among other ratios (33\% oil uptake \%). Oil to potato 10:1 had the lowest amount of fat content (24\% oil uptake \%). By this study, the highest and lowest loss weight percent were $44 \%$ and $34 \%$ which related to ratio 20 and 10 respectively. Significant differences in texture of several ratio were observed. Ratio 20 was the most texture of fried potato, but from view of oil uptake \%, ratio of 10 was the best for two sizes of French fries potato.
\end{abstract}

Volume I Issue I - 2015

\author{
Habib O Mirzaei,' Frank Farhadpour² \\ 'Aristotle University of Thessaloniki, Greece \\ ${ }^{2}$ Master of food science and technology, Greece
}

Correspondence: Habib O Mirzaei, Aristotle University of Thessaloniki, no I33.olibidoze street, Greece, Tel +306951396398, Email mirzaeihabib I@gmail.com

Received: May 19,2015 | Published: May 28, 2015

Keywords: ratio of oil in potato, oil uptake, French fries

\section{Introduction}

In the french fry process, raw potato are washed and cut into strips after this potato strips are fried in hot oil $\left(180^{\circ} \mathrm{C}\right)$, cooled in ambient air and finally frozen and packaged on the other hand, air dehydration leads to a lower initial moisture content which also reduces the oil absorption. One way to achieve that is by controlling of frying temperture, ratio oil to potato etc. The main goal of deep fat frying is to maintain the food flavor inside a tenderized and crispy crust. Quality attributes in fried products includes dry matter, oil content, color and texture. Oil content is one of the important quality attributes in fried products. ${ }^{1}$ Today consumers are looking for food products with lower oil content, therefore it is important to find the ways that can reduce the oil content of fried products. During the frying process, the physical, chemical and sensory characteristics of the food are modified. Texture, color and oil content are the main characteristics or quality parameters of French fries. Good quality French fries must have a crispy of about $1-2 \mathrm{~mm}$ where most of the oil is located and a wet, soft center, like a cooked potato. ${ }^{2}$ French fries color is the result of maillard reaction that depends on the superficial content of reducing sugar and temperture and frying period. Another way to achieve that is by controlling ratio oil to potato, because higher ratio leads to higher absorbed oil. ${ }^{3,4}$ Oil content has been a main concern for food processors from an economic point of view and for consumers from a healthy one. During the process of French fries manufacture, processors have some ways to control the oil picked up by the potato pieces, as explained below. Today, due to consumer health concerns, there is a strong incentive to reduce the oil content of fried foods. . $^{5,6}$ Some fried products may contain fat up to $50 \%$ of the total weight. Some of these lipids were not in the food before frying. Lipid content of French fries increases from $2 \%$ to $14 \%$. The objective of the present work was to apply ratio oil to potato for reduce oil uptake in French fries potato.

\section{Materials and methods}

\section{Sample preparation}

Potato were purchased from Greece market, after peeling, they were converted with two shapes were deep fried at $180^{\circ} \mathrm{C}$ for 10 minute.

\section{Experiments}

Changes of weight of potato, temperture of oil with time of frying were plotted, thickness of crust and thermal conductivity coefficient (K) were measured. These experiments were done for ratios oil to potato 20,10 separately.

a. Sunflower oil was supplied from local market of Greece.

b. Strip of potato were prepared with two dimension $3 \mathrm{~cm}$ (diameter) and thickness of $1 \mathrm{~cm}$, other size were $1 \mathrm{~cm}$ of length at $0.5 \mathrm{~cm}$ of diameter at $0.5 \mathrm{~cm}$ of thickness

\section{Statistical analysis}

All these experiments were replicated 3 times and the average values were reported. Significant differences of means were compared using Duncan test at 5\% significant level using suitable software program.

\section{Results and discussion}

We found for two sizes of French fries potato that there was significant difference $(\mathrm{p}<0.05)$ among two ratio oil to potato in items of $\%$ loss water (Figure 1). The highest $\%$ loss water was ratio 20 and the lowest was ratio 10 , our results revealed that percent of oil at ratio 20 and 10 were $33 \%$ and $24 \%$ respectively. As it can be seen in Figure 1, loss weight of potato at time 10 minute was $42 \%$ and $34 \%$ for ratio 20 and 10 respectively. This different were significant 
$(\mathrm{p}<0.05)$. Temperture of oil were after 10 minute of frying $187^{\circ} \mathrm{C}$ and $178^{\circ} \mathrm{C}$ for ratio $20: 1$ and $10: 1$ respectively and this different was significant $(\mathrm{p}<0.05)^{7}$ (Figure 2). Considering thermal conductivity coefficient analysis were observed difference significant between ratios 20 and $10(\mathrm{p}<0.05)$. That valves were 620 and $550 \mathrm{wat} / \mathrm{c} \mathrm{sec}$ for ratios 20 and 10 respectively (Figure 3 ). Results showed thickness of crust were significant for ratios that values were 3 and $1,2 \mathrm{~mm}$ for ratio 20 and 10 respectively $^{8}$ (Figure 4 ). At last, oil uptake \% were 33 and 24 for ratio 20 and 10 respectively thus ratio 10 was suitable for frying of potato $(\mathrm{p}<0.05)$ (Figure 5$)$.

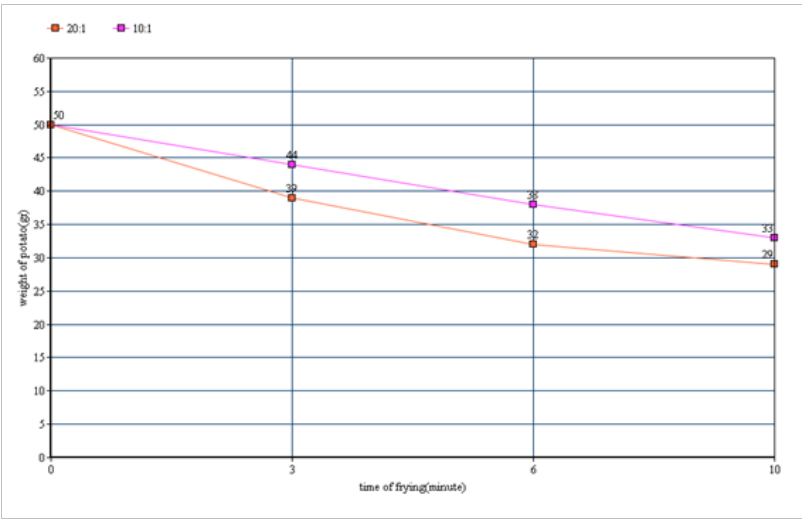

Figure I Some properties of French fries potato.

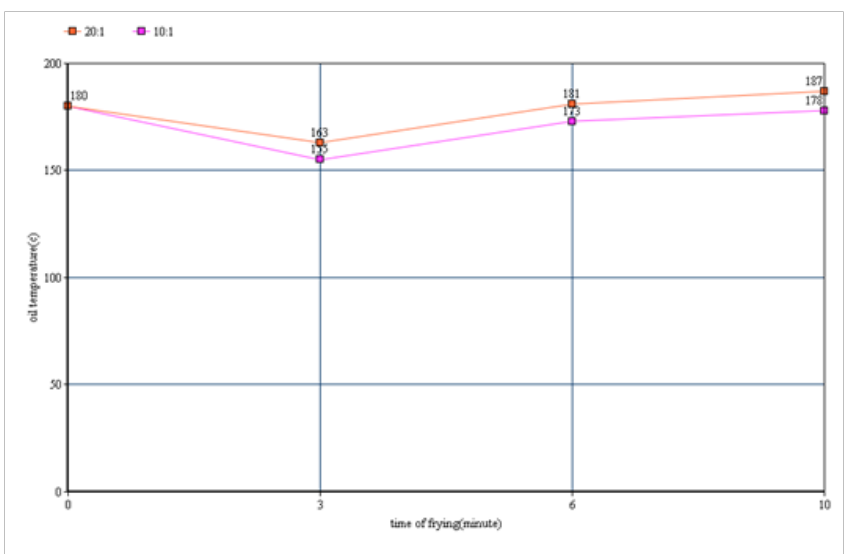

Figure 2 Some properties of French fries potato.

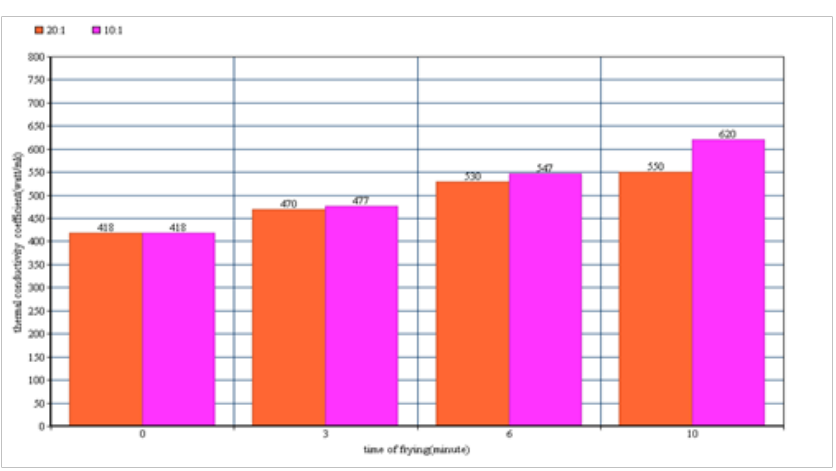

Figure 3 Some properties of French fries potato.

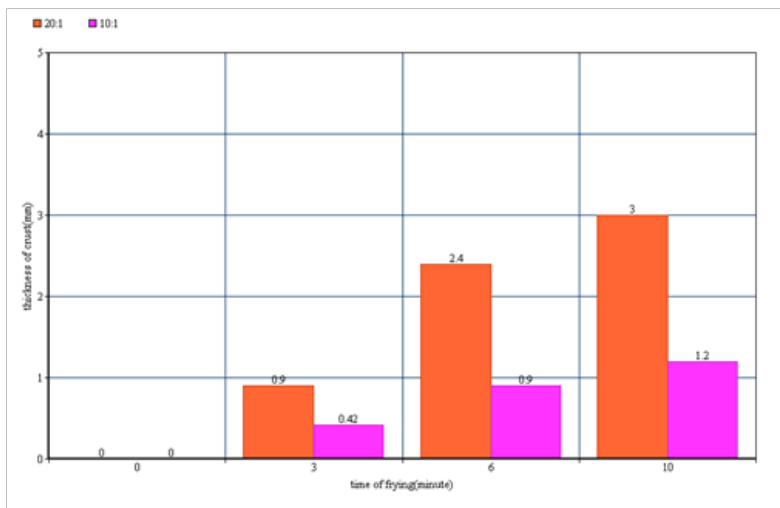

Figure 4 Some properties of French fries potato.

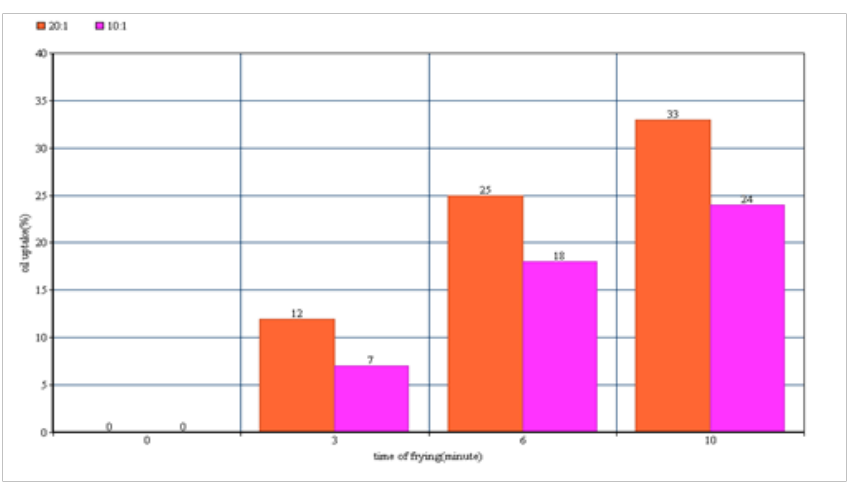

Figure 5 Some properties of French fries potato.

\section{Conclusion}

Our results showed that oil uptake were at ratio 10 less than ratio 20 , thickness of crust were significant difference between ratios 20 and $10(p<0.05)$. By considering, the best results of oil uptake were ratio oil to potato 10 .

\section{Acknowledgements}

None.

\section{Conflict of interest}

The author declares no conflict of interest.

\section{References}

1. García M, Bifani V, Campos C, et al. Edible coating as an oil barrier or active system. Journal of food engineering. 2008;57:225-241.

2. Saguy I, Karel M. Modeling of quality deterioration during food processing and storage. Food technology. 1980;34(2):78-85.

3. Moyano PC, Ríoseco VK, Gonzaléz PA. Kinetics of crust color changes during deep-fat frying of impregnated French fries. J Food Eng. 2002;54(3):249-255.

4. Alava M, Dubé M, Rost M. Imbibition in disordered media. Adv Phys. 2004;53(2):83-175.

5. Moreira RG, Castell-Pérez ME, Barrufet MA. Deep-Fat Frying: Fundamentals and Applications. An Aspen Publication, Gaithersburg, Maryland. USA: Aspen Publishers, Inc; 1999. p. 3-74. 
6. Ni H, Datta AK. Moisture, oil and energy transport during deep fat frying of food material. Food and bioprocess processing. 1999;77(3):194-204.

7. Williams R, Mittal GS. Low fat fried foods with edible coating. Journal of Food Science. 19997;64(2):317-322.
8. Ateba P. Dynamics of crust formation and kinetics of quality changes during frying of Meatballs. Journal of food science. 1994;59(6):12751278 